\title{
Publisher's Note: Amplitude-phase coupling drives chimera states in globally coupled laser networks [Phys. Rev. E 91, 040901(R) (2015)]
}

Fabian Böhm, Anna Zakharova, Eckehard Schöll, and Kathy Lüdge (Received 9 December 2015; published 28 December 2015)

DOI: 10.1103/PhysRevE.92.069905

This paper was published online on 22 April 2015 with an error in Eq. (1). Equation (1) should read as

$$
\frac{d E_{n}}{d t}=(1+i \alpha) E_{n} N_{n}+e^{-i C_{p}} \kappa \sum_{j}^{Z} e^{-i C_{p}} E_{j}(t-\tau)
$$

The equation has been corrected as of 9 December 2015. The equation is incorrect in the printed version of the journal. 